\title{
Low cost fabrication of microfluidic paper-based analytical devices with water-based polyurethane acrylate and their application for bacterial detection
}

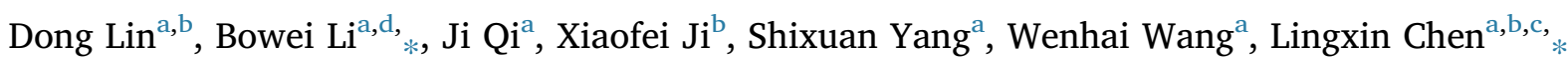 \\ ${ }^{a}$ CAS Key Laboratory of Coastal Environmental Processes and Ecological Remediation, Research Center for Coastal Environmental Engineering and Technology, Yantai \\ Institute of Coastal Zone Research, Chinese Academy of Sciences, Yantai 264003, China \\ ${ }^{\mathrm{b}}$ School of Pharmacy, Binzhou Medical University, Yantai 264003, China \\ ${ }^{\mathrm{c}}$ Laboratory for Marine Biology and Biotechnology, Pilot National Laboratory for Marine Science and Technology, Qingdao 266237, China \\ ${ }^{\mathrm{d}}$ Center for Ocean Mega-Science, Chinese Academy of Sciences, Qingdao 266071, China
}

\section{A R T I C L E I N F O}

\section{Keywords:}

Paper microfluidics

muPAD

Polyurethane acrylate

Ultraviolet light curing

Bacterial detection

\begin{abstract}
A B S T R A C T
This study presents a simple, inexpensive and environment-friendly fabrication strategy for microfluidic paperbased analytical devices which can resist the penetration of surfactant solutions and organic solvents, by using water-based polyurethane acrylate via UV light curing. The filter paper's barrier created using cured PUA could withstand surfactant solutions (10 wt \%, CTAB, SDS and Triton X-100) and organic solvents (methanol, isopropanol, DMF, DMSO, etc). This is very useful for analyzing complicated biological samples on the microfluidic paper-based analytical devices. In addition, the expense of water-based polyurethane acrylate is very cheap (about $\$ 8 / 500 \mathrm{~g}$ ) and PUA developer is water that is environmental-friendly. To further verify its advantage, we successfully demonstrated the proposed microfluidic devices for detection of $E$. coli targets in tap water and seawater via colorimetric analysis in a fast and convenient manner. Our results revealed that the linear response to $E$. coli BL21 was in the range of $10^{4} \sim 10^{9} \mathrm{cfu} / \mathrm{mL}$. The proposed method can effectively avoid the damage for the hydrophobic barriers from the solution even some aggressive liquids, and shows great potential in on-site analysis, environmental monitoring, and food safety.
\end{abstract}

\section{Introduction}

Microfluidic paper-based analytical devices ( $\mu$ PADs) have attracted considerable interest in qualitative or quantitative assays because of their low cost, portability, biocompatibility and high efficiency [1-6]. Nowdays, $\mu$ PADs as analysis platforms, have developed rapidly in electrochemical analysis [7-9], environmental monitoring [10-14], clinical diagnostics $[15,16]$ and food analysis $[17,18]$.

Since the first report from Whitesides group patterned chromatography paper using photolithography $[19,20]$, a series of methods have been proposed for fabrication of $\mu$ PADs by partially hydrophobizing the filter paper, including wax printing and dipping [21-24], inkjet printing [25], screen printing [26], vapor phase deposition [27], plasma treatment [28,29], laser cutting [30,31] and hand drawing [32]. Although more and more materials and technologies for fabrication of $\mu$ PADs have developed, each has its own advantages and limitations. Wax printing is the mostly popular method based on its simplicity, universality, economy and suitability for mass production [21]. Unfortunately, the hydrophobic barriers based this material could be penetrated by low surface tension surfactant solutions and organic solvents which commonly used in bioanalysis. In addition, the resolution of generated hydrophilic channels was low because the wax would not only penetrate through the filter paper vertically but also flow laterally during the baking. SU-8 photolithography provided hydrophobic barriers can effectively resist surfactant solutions and organic solvents, but this method usually requires expensive commercialized SU-8 photoresist and complicated fabrication process [19]. Bruzewicz et al. developed a PDMS plotting method to define channels on paper [33]. The $\mu$ PADs fabricated by this method had excellent flexibility and relatively low cost, but the resolution of hydrophilic channels was also low. Wang et al. utilized hydrophobic sol-gel derived methylsilsesquioxane (MSQ) as barrier materials defining channels by direct inkjet printing of the barriers on paper and successfully withstood surfactant solutions and organic solvents [34]. However, its fabrication required

\footnotetext{
* Corresponding authors at: CAS Key Laboratory of Coastal Environmental Processes and Ecological Remediation, Research Center for Coastal Environmental Engineering and Technology, Yantai Institute of Coastal Zone Research, Chinese Academy of Sciences, Yantai 264003, China.

E-mail addresses: bwli@yic.ac.cn (B. Li), lxchen@yic.ac.cn (L. Chen).
} 

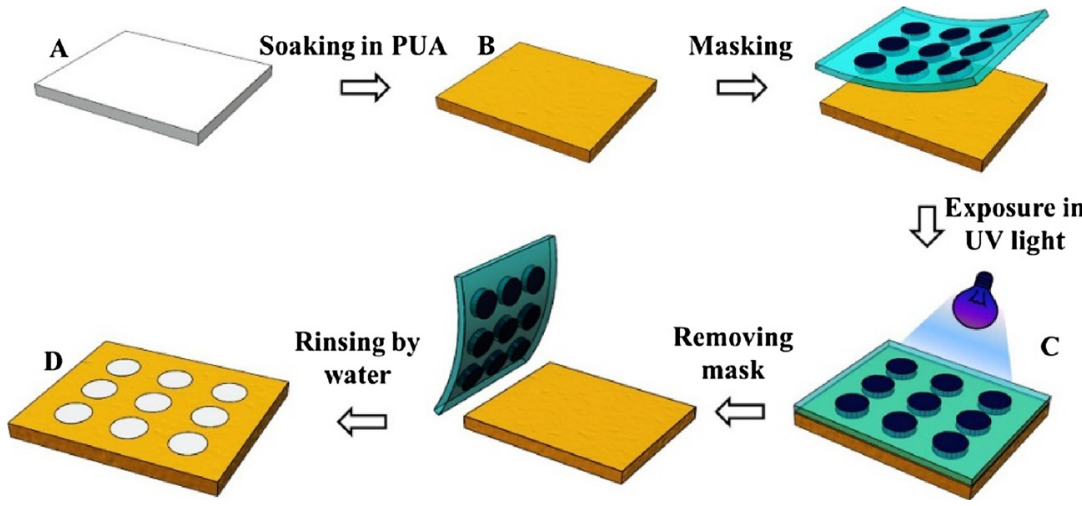

Pure filter paper
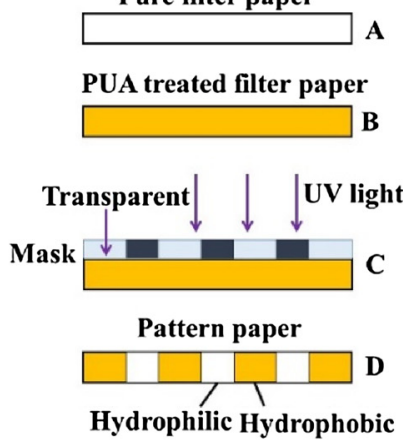

Fig. 1. Schematic representation of process to fabricate $\mu$ PADs using water-based PUA through UV light curing.

first 3 printing passes of Tris buffer and then pringting three layers of MSQ precursor ink. The process was cumbersome and time-consuming. Furthermore, the position of the printed hydrophobic region may change during printing 6 times. In addition, the condensed MTMS sol could clog the cartridges at printing process. Nurak et al. reported a simple fabrication approach for $\mu$ PADs via spraying the acrylic lacquer onto paper to create hydrophobic barriers, but the acrylic lacquer contained volatile organic compounds (VOC), which were detrimental to human health [35]. In addition, the acrylic lacquer lacks flexibility, causing the hydrophobic barriers to be fragile. Cardoso et al. developed a spraying method using scholar glue to fabricating paper-based devices [36]. This method required only a spray system and a UV lamp. However, the pattern structures in paper only provided similar size resolution as wax printing. Derda et al. described a simple approach for the patterned deposition of Teflon on paper to prepare an integrated platform for parallel organic synthesis and cell-based assays. The developed platform significantly improved the peptide yield and successfully used for screening breast cancer cells [37]. With the increasing use of $\mu$ PADs in the bioanalysis field, it is necessary to find a simple, inexpensive and environment-friendly strategy for fabricating $\mu$ PADs which can effectively resist the penetration of surfactant solutions and organic solvents.

As a novel photosensitive resin, polyurethane acrylate (PUA) is developing rapidly based on its excellent performance in recent years. This material not only has the flexibility and abrasion resistance of polyurethane but also has the chemical resistance and transparency of acrylate, and has been widely used in coating, screen printing inks, paper glazing coating and other industrial fields [38-41]. Fonseca and colleagues reported the construction of a microfluidic polymer chip in PUA resin [42-44]. However, to our best knowledge, it was the first time that PUA was used to fabricate $\mu$ PADs.

Herein we described a novel method for fabricating $\mu$ PADs by patterning the filter paper using water-based PUA via UV light curing. The ability for resisting surfactant solutions and organic solvents of the $\mu$ PADs was investigated. Furthermore, the utility of the $\mu$ PADs was demonstrated by colorimetric assay of E. coli BL21 in tap water and seawater. The proposed $\mu$ PADs can provide an effectively tool for rapid, simple and cost-effective detection of bacteria in the field.

\section{Materials and methods}

\subsection{Reagents and materials}

Whatman No. 1 filter paper was obtained from Hangzhou Xinhua Paper Limited Company (GE Healthcare, China). Water-based PUA containing prepolymer with a mass fraction of $45 \%$ was purchased from Nanjing Jiazhong Chemical Technology Co., Ltd. (Nanjing, China). photoinitiator 2-hydroxy-2-methylpropiophenone (HMPP) and polyvinylamine (PVAm) were from BASF. Methanol, isopropanol, N, NDimethylformamide (DMF), dimethyl sulfoxide (DMSO), sodium dodecylsulfate (SDS) and toluene were from Sinopharm Chemical Reagent (Shanghai, China). Chlorophenol red- $\beta$-D-galactopyranoside (CPRG), Triton X-100 and cetyltrimethylammonium bromide (CTAB) were purchased from Sigma-Aldrich. The masks (polyethylene terephthalate substrate) with the designed patterns were obtained from Dalian Qinling Digital Printing Technology Co., Ltd (Dalian, China). B$\mathrm{PER}^{\circledast}$ direct bacterial protein extraction reagent was purchased from Thermo Scientific. All the chemicals were used as received without further purification. Deionized water produced by a Pall Cascada laboratory water system (g18.2 M 2 , Millipore, Bedford, MA, USA) was used in all solutions and assays.

\subsection{Fabrication of $\mu P A D s$}

The filter paper was cut in a desired size and then immersed in a mixed solution of water-based PUA and photoinitiator HMPP (3\% of the total mass of PUA formulation. Firstly, HMPP was added to PUA solution and then stirred evenly) for $1 \mathrm{~min}$. After adsorbed the solution, the filter paper was baked in a vacuum drying oven at $80^{\circ} \mathrm{C}$ for $10 \mathrm{~min}$. Then, the surface of the filter paper was covered by the mask with the designed patterns (Fig. S1). The assembly was then exposed by a UV lamp (LED, $395 \mathrm{~nm}, 15 \mathrm{~mW} / \mathrm{cm}^{2}$ ) for $5 \mathrm{~min}$. After exposure, the filter paper was rinsed with water for $1 \mathrm{~min}$ to remove uncured PUA and then the expected $\mu \mathrm{PAD}$ was obtained after dry. The fabrication process was schematically shown in Fig. 1 and the actual fabrication process was shown in Fig. S2.

\subsection{Assay of E. coli targets}

Assay of E. coli BL21was carried out in accordance with the previously described method [45]. Briefly, $10 \mu \mathrm{L}$ polyvinylamine (PVAm, $0.5 \mathrm{wt} \%$ in water) was firstly manually spotted inside the hollowed circles ( $7 \mathrm{~mm}$ outer diameter, $6 \mathrm{~mm}$ inner diameter), followed by $10 \mu \mathrm{L}$ CPRG (3 mM in water) as colorimetric substrates. E. coli BL 21 was grown in tryptic soy broth with yeast extract (TSB-YE) media at $37^{\circ} \mathrm{C}$ with gentle shaking at $125 \mathrm{rpm}$ for $24 \mathrm{~h}$. Serial dilutions of bacterial suspensions were prepared in the range from $10^{1}$ to $10^{9} \mathrm{cfu} / \mathrm{mL}$ in TSBYE. The numbers of colony-forming units per milliliter ( $\geq 10$ bacteria per sample) were counted using a standard plate counting method. The bacterial samples were then lysed using ${\mathrm{B}-\mathrm{PER}^{\circledR}}^{\circledR}$ at 9:1 vol ratio (bacterial samples: B-PER ${ }^{\circledast}$ reagent) following the supplier's protocol. $10 \mu \mathrm{L}$ of bacteria lysate was dropped onto the colorimetric substrates of the hollowed circles. After $10 \mathrm{~min}$, the colour intensity was measured by our in-house made device (Fig. S3) [46]. All experiments with bacteria in this work were implemented in a bio-safety level 2 (BSL-2) laboratory. 


\section{Results and discussion}

\subsection{Fabrication of $\mu P A D s$}

Fabrication of $\mu$ PADs using water-based PUA via UV light curing has some advantages (Table S1). Compared with SU-8 photolithography and MSQ printing, this method is much simpler and environmentfriendly. The fabrication process does not require expensive equipment and complicated steps. Moreover, the price of water-based PUA is very cheap $(\$ 8 / 500 \mathrm{~g})$ and the cost for fabricating an 8.5 in $\times 11$ in sheet of $\mu \mathrm{PAD}$ using the proposed method was only about $\$ 0.8$. In addition, water-based PUA uses water as a solvent without any toxic VOC and uncured PUA can also be easily rinsed down with water, hence the fabrication process is quite environment friendly. It was also found from Table S1 that the wax printing method was still the fastest and lowest cost, but the wax based barriers could not resist some aggressive liquids [34].

\subsection{Optimization of fabrication conditions}

The filter paper becomes hydrophobic due to the crossing-link of PUA adsorbed on the paper fiber resulted from the radical polymerization between the $\mathrm{C}=\mathrm{C}$ in different PUA molecules under UV light. Therefore, the PUA concentration and the exposure time are two key factors for fabrication of $\mu$ PADs.

The filter paper was treated with different concentrations of PUA solution. 20, 30 and 40\% PUA were obtained by diluting original PUA (containing prepolymer with a mass fraction of 45\%) with water. And $50 \%$ PUA was obtained by concentrating original PUA using rotary evaporation. $20 \mathrm{~g}$ of $45 \%$ PUA was weighted and evaporated to $18 \mathrm{~g}$, and its final concentration was $50 \%$. Fig. 2 A showed the SEM micrographs of PUA treated filter paper with different concentrations of PUA solution. It was found the untreated filter paper had lots of pores. By contrast, the porosity of the filter paper decreased with the increasing of PUA concentration and the surface of the filter paper became smooth with little porosity when the concentration increased to $45 \%$. Microfluidic barriers can work either by transforming cellulose surfaces to being non-wetting or by filling the pores with an insoluble material [34]. Obviously, our proposed methodology belongs to the latter. Different concentrations of PUA were used to fabricate hollowed circles ( $6 \mathrm{~mm}$ outer diameter, $5 \mathrm{~mm}$ inner diameter) and then dyed blue color water was dropped onto them. The photos were recorded with a Cannon 600D camera. As shown in Fig. 2B, with the increasing of PUA concentration, the profile of the hydrophobic barriers became clearer. When the concentration of PUA was $45 \%$, the dyed color water could not penetrate out of the hollowed circles. Further increasing of the PUA solution concentration to $50 \%$ would prolong the followed exposure time and raise the cost, but the surface morphology and waterproof performance of the filter paper didn't change significantly (Fig. 2A (f)). Therefore, we selected the optimal condition as 45\% PUA.

The exposure time would have an influence for the structure of hydrophobic barriers because the uncompleted cross-linking of PUA could be dissolved by solutions in some degree. During testing, the degree of cross-linking of PUA could improve with increasing of the exposure time. After $5 \mathrm{~min}$, the data (not shown) illustrated that PUA had completely crosslinked and could resist the penetration by water.

\subsection{Performance characterization of the propose $\mu$ PADs}

In order to evaluate the performance of containing water solution of the $\mu$ PADs, dyed water was dropped onto the hydrophobic and hydrophilic regions of the patterned filter paper, respectively. As shown in Fig. 3A, the dyed red color water formed a droplet in the hydrophobic region with an apparent static contact angle $116^{\circ}$ (Fig. 3C), while immersed into the entire hydrophilic region quickly. As shown in Fig. 3B, the dyed water was perfectly confined in the different hydrophilic patterns without penetrating into the hydrophobic region. The results indicated that the $\mu$ PADs with various hydrophilic channels could be fabricated using the proposed method.

The resolution of hydrophilic-hydrophobic patterns on the fabricated $\mu$ PADs was also characterized. A series of microfluidic channels and hydrophobic barriers with different dimensions (from 600 to $50 \mu \mathrm{m}$, in decrement of $50 \mu \mathrm{m}$ ) were patterned on the filter paper. Firstly, the dye orange color water was dropped onto the middle rectangle reservoir, and then spread under capillary action. The widths of microfluidic channels and hydrophobic barriers were measured using a digital microscope (Olympus DSX510). Fig. 3D showed the thinnest microchannel that the dye water could completely pass was $200 \mu \mathrm{m}$ in designed width and was measured to be $180 \pm 20 \mu \mathrm{m}(\mathrm{n}=5)$. Fig. $3 \mathrm{E}$ showed the thinnest hydrophobic barriers which could confine the dye water was $200 \mu \mathrm{m}$ in designed width and was measured to be $200 \pm 20 \mu \mathrm{m}(\mathrm{n}=5)$. The resulted resolution was comparable to that of the $\mu$ PADs fabricated by SU-8 photolithography (Table S1).

Surfactant solutions and organic solvents are commonly used in bioanalysis. However, wax printed barriers could be destroyed by these reagents, which limited their versatile applications. In order to test the chemical resistance of the PUA based barriers, $10 \mu \mathrm{L}$ surfactant solutions with different concentration (CTAB, SDS and Triton X-100) and organic solvents (methanol, isopropanol, DMF, DMSO and toluene) were dropped inside the PUA based hollowed circles $(6 \mathrm{~mm}$ outer diameter, $5 \mathrm{~mm}$ inner diameter). A comparative study was performed using the wax printed hollowed circles, which were printed by an 8560DN wax printer (Fuji XEROX Company). As shown in Fig. 4A, the wax based barriers were quickly breached by all of the three surfactant solutions ( $1 \mathrm{wt} \%)$. By contrast, the PUA based barriers were not breached by these surfactant solutions. Even if the concentrations of the three surfactant solutions were increased to $10 \mathrm{wt} \%$, the PUA based barriers would not be breached (Fig. S4). For organic solvents test (Fig. 4B), the wax-based barriers could not resist any of the chose organic solvents. However, the PUA based barriers were able to resist methanol, isopropanol, DMF and DMSO, only breached by toluene with weak polarity. This is because the pores in the filter paper are filled with crosslinked PUA which is hardly penetrated by surfactant solutions and organic solvents, and the capillary action are disabled. Hence, our proposed $\mu$ PADs are more suitable for the bioanalysis than the $\mu$ PADs prepared by wax printing method due to their good chemical resistance.

\subsection{Assay of E. coli BL21}

Previous reports have used ultrasonic lysis in bacterial extraction because the surfactants in the B-PER ${ }^{\circledast}$ can destroy the wax based hydrophobic region and causes leakage [45]. Our approach can effectively solve this problem and to further validate our proposed method, a series of $E$. coli BL21 samples with concentrations ranging from $10^{1}$ to $10^{9}$ $\mathrm{cfu} / \mathrm{mL}$ were investigated. The detection of $E$. coli BL21 was performed by measuring the color change when intracellular enzyme $\beta$-galactosidase reacting with a chromogenic substrate CPRG. In the presence of $\beta$ galactosidase, the color of CPRG can change from yellow to red-violet. Prior to colorimetric detection, E. coli BL21 samples were lysed by BPER $^{\circledast}$ (which contains surfactant component and commonly used for bacterial lysis) to release $\beta$-galactosidase. Because $\beta$-galactosidase was also present in dead E. coli BL21, the total amounts of E. coli BL21 (including living and dead bacteria) was detected using this method. Fig. 5A showed the colorimetric assay for E. coli BL21 $\left(\sim 10^{9} \mathrm{cfu} / \mathrm{mL}\right)$ using the PUA based circles and the wax printed circles. it was observed that the bacterial lysate penetrated outside the wax printed circle to cause the leakage and the analysis result was seriously interfered. Meantime, the E. coli BL21 lysate was well confined inside the PUA based circles with an obvious color change. This result showed that PUA was well resistant to the destruction of surfactant component in B$\mathrm{PER}^{\circledR}$ and demonstrated the potential application in bioanalysis.

To further validate the applicability of this proposed method for 

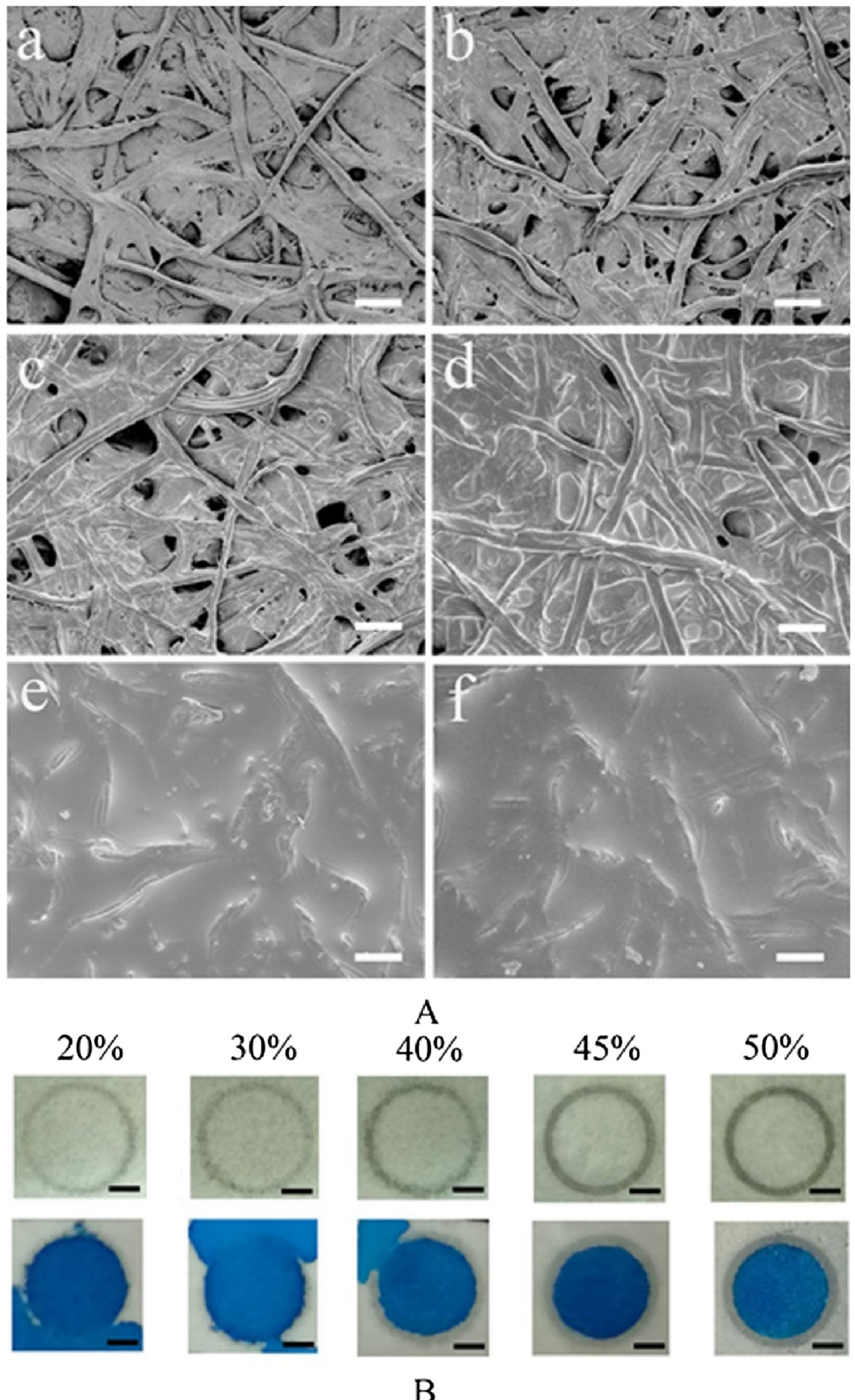

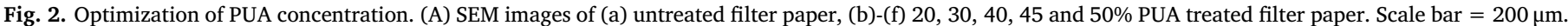

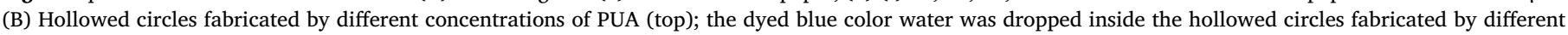
concentrations of PUA (bottom). Scale bar $=3 \mathrm{~mm}$.

quantitative measurement of bacteria, a series of E. coli BL21 suspensions with different concentrations were detected on the PUA based $\mu$ PADs. As shown in Fig. 5B, the intensity of the color was linearly dependent upon the concentration of $E$. coli BL21 with a linearity range from $10^{4}$ to $10^{9} \mathrm{cfu} / \mathrm{mL}$. The linear correlation coefficient $\mathrm{R}^{2}$ was 0.965 with a detection limit of $3.7 \times 10^{3} \mathrm{cfu} / \mathrm{mL}$, which was comparable to those of commercialized field-based $E$. coli detection methods: MaxSignal $^{\circledast}$, RapidChek ${ }^{\circledast}$, Gen-Probe $^{\circledast}$, IQuum $^{\circledast}$ and Watersafe ${ }^{\circledR}$ [47]. Fig. S5 


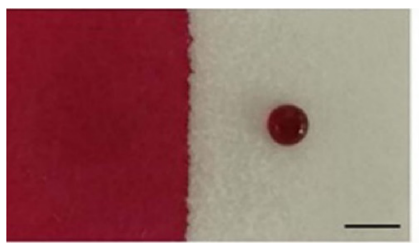

A

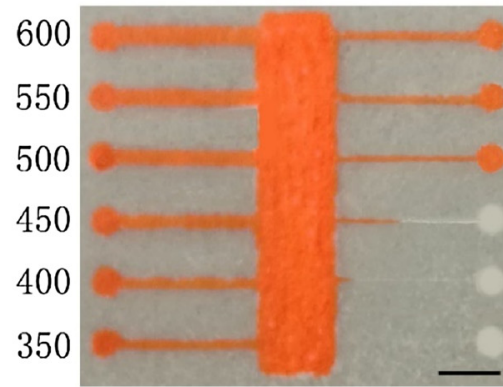

D

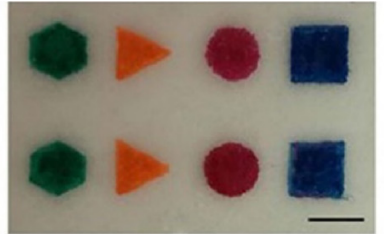

B

300
250
200
150
100
50

(1)

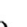

300
250
200
150
100
50

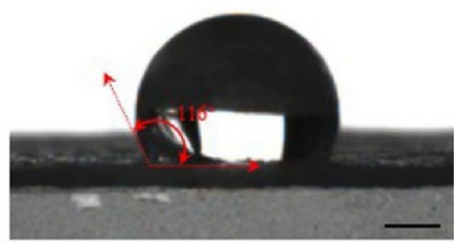

C

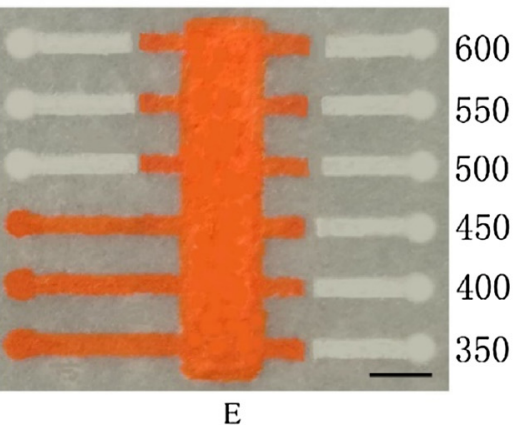

Fig. 3. Characterization of the PUA patterned filter paper. (A) $5 \mu \mathrm{L}$ dye water was dropped onto the unexposed region (left side) and the exposed region (right side), respectively. (B) Different colored dye water was added on the hydrophilic pattern. (C) Water contact angle of the hydrophobic region was $116^{\circ}$. (D) The resolution of the hydrophilic microchannels. (E) The resolution of the hydrophobic barriers. Scale bar $=4 \mathrm{~mm}$. showed the reliability of colorimetric detection of E. coli BL21 with different concentrations $\left(10^{5}, 10^{6}, 10^{7}\right.$ and $\left.10^{8} \mathrm{cfu} / \mathrm{mL}\right)$ using the proposed $\mu$ PADs. The RSD of colour intensity were $3.15 \%, 3.62 \%$, $2.26 \%$ and $2.78 \%$, respectively, which indicated that the proposed $\mu$ PADs possessed good reproducibility.

\subsection{Detection of E. coli BL21 from inoculated water sample}

To demonstrate proof-of-concept for the feasibility of the $\mu$ PADs to detect bacteria in the field, $E$. coli BL21 detection was implemented in two types of water samples. Tap water samples were obtained from our

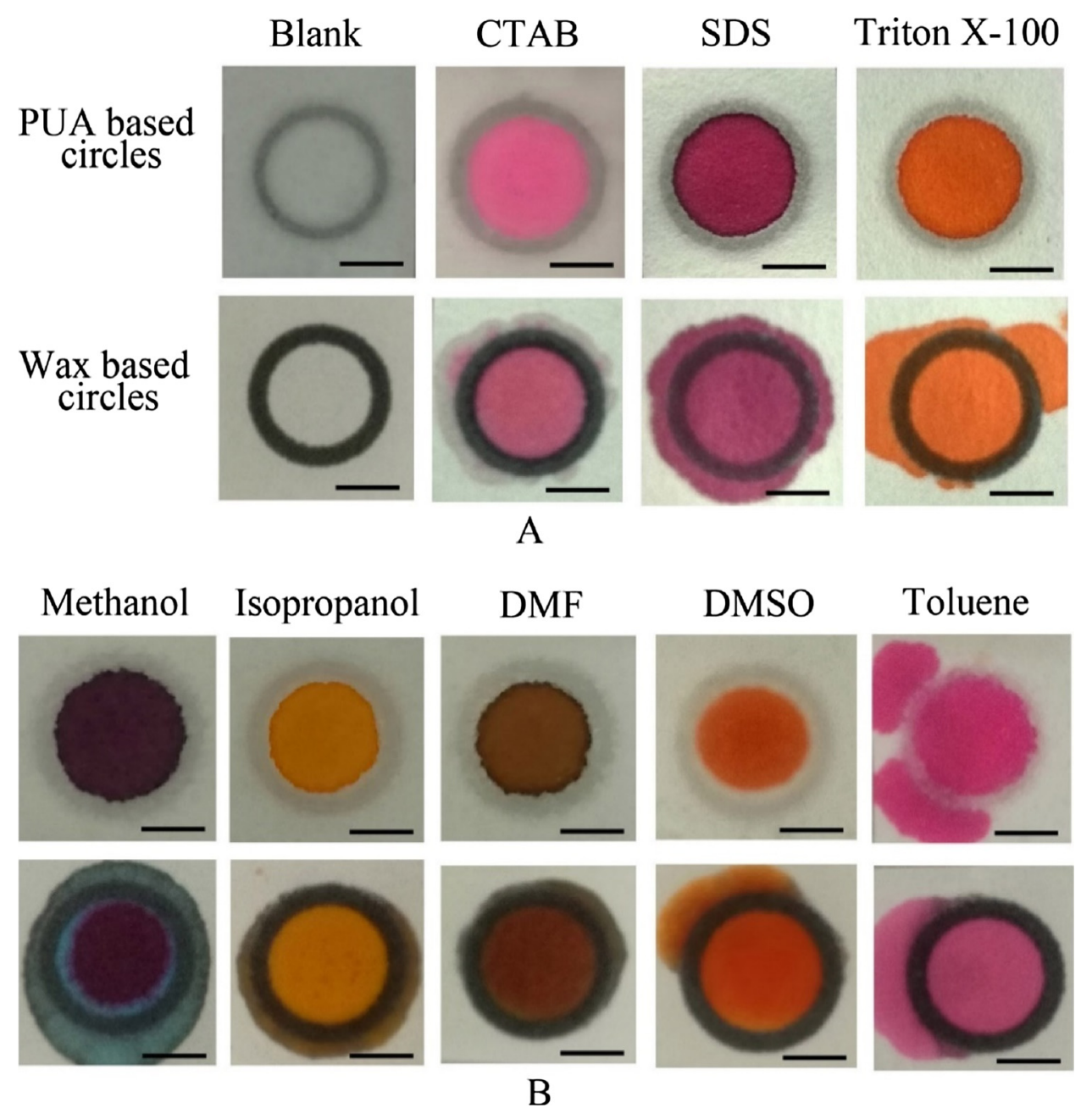

Fig. 4. Comparison of the PUA based barriers and the wax printed barriers for resisting the surfactant solutions (A) and organic solvents (B). The concentration of all three surfactants is $1 \mathrm{wt} \%$. Scale bar $=3 \mathrm{~mm}$. 


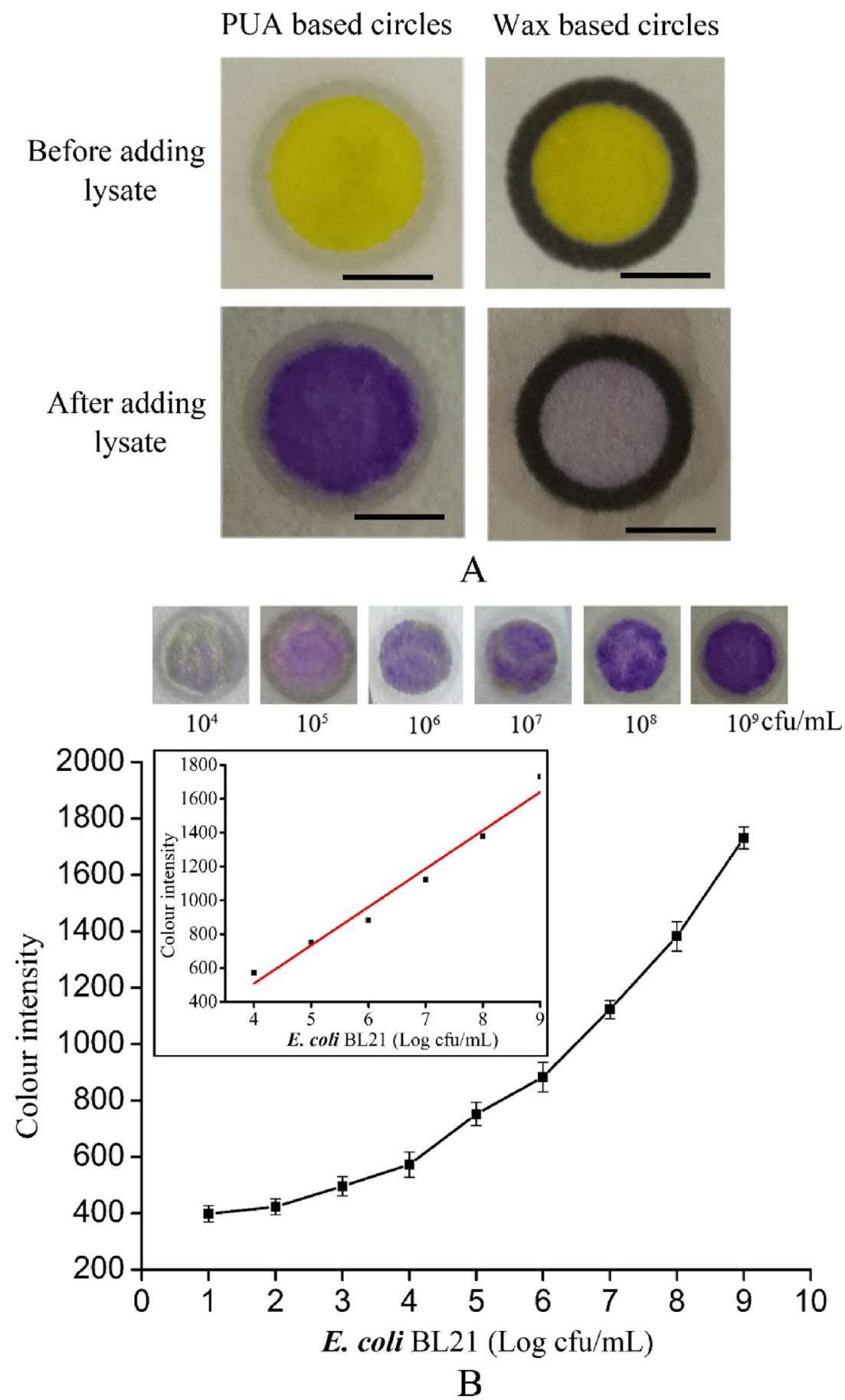

Fig. 5. Detecting of E. coli BL21. (A) Optical micrographics showing the colorimetric assays for E. coli BL21 using the PUA based circles and the wax printed circles. (B) Detection of $E$. coli BL21 with increasing concentrations using the PUA based circles. For each concentration, the values are the average of three replicates. Scale bar $=5 \mathrm{~mm}$.

Table 1

Recovery of spiked E. coli BL21 into tap water and seawater.

\begin{tabular}{lllll}
\hline Sample & $\begin{array}{l}\text { Added (cfu/ } \\
\mathrm{mL})\end{array}$ & $\begin{array}{l}\text { Detected (cfu/ } \\
\mathrm{mL})\end{array}$ & $\begin{array}{l}\text { Recovery } \\
\mathrm{RSD}(\%)\end{array}$ & $\begin{array}{l}\text { Standard plating } \\
\text { results }\end{array}$ \\
\hline Tap water & $1.0 \times 10^{4}$ & $1.04 \times 10^{4}$ & $104.0 \pm 3.4$ & $0.97 \times 10^{4}$ \\
& $2.5 \times 10^{5}$ & $2.37 \times 10^{5}$ & $94.8 \pm 3.9$ & $2.42 \times 10^{5}$ \\
& $5.0 \times 10^{6}$ & $4.86 \times 10^{6}$ & $97.2 \pm 4.3$ & $4.83 \times 10^{6}$ \\
Seawater & $1.0 \times 10^{4}$ & $0.93 \times 10^{4}$ & $93.0 \pm 4.8$ & $0.95 \times 10^{4}$ \\
& $2.5 \times 10^{5}$ & $2.57 \times 10^{5}$ & $102.8 \pm 4.5$ & $2.43 \times 10^{5}$ \\
& $5.0 \times 10^{6}$ & $5.34 \times 10^{6}$ & $106.8 \pm 3.6$ & $4.96 \times 10^{6}$ \\
\hline
\end{tabular}

\footnotetext{
average value from three individual experiments.
}

office, and seawater samples were taken from the coastal zone of the Yellow Sea of China. The spiked concentrations of $E$. coli BL21 were $1.0 \times 10^{4}, 2.5 \times 10^{5}$ and $5.0 \times 10^{6} \mathrm{cfu} / \mathrm{mL}$. As listed in Table 1 , the recoveries were attained of $94.8-104.0 \%$ with RSDs of $3.4-4.3 \%$ for tap water, and $93.0-106.8 \%$ with RSDs of $3.6-4.8 \%$ for sea water. The results indicated that such a method was practically feasible for determination of $E$. coli BL21 in different environmental water samples. Standard plating method was also implemented to confirm the concentration of $E$. coli BL21 spiked into tap water and seawater samples. The results from plating matched the results from the results from the proposed $\mu$ PADs, but standard plating method took at least $48 \mathrm{~h}$ to 
make a count of the numbers of colony-forming units.

\section{Conclusions}

In this study, we firstly presented the strategy to fabricate the $\mu$ PADs by using water-based PUA through UV light curing. The fabrication process is simple and environment-friendly and does not require expensive equipment and reagents. The PUA based hydrophobic barriers are not only can contain aqueous solutions, but also can effectively resist the damages by many surfactant solutions and organic solvents that can destroy the wax based hydrophobic barriers. This advantage will play a significant role especially in the detection of biological samples. To further verify the performance of the proposed method, $E$. coli BL21 was used as a model target and implemented colorimetric detection on the fabricated $\mu$ PADs with the limit detection of $3.7 \times 10^{3}$ $\mathrm{cfu} / \mathrm{mL}$. The proposed $\mu$ PADs exhibit great potential and considerable versatility for detecting different targets. We believe that this facile and low-cost approach has a broad application prospect in the field of onsite analysis, environmental monitoring and food safety in the future.

\section{Declaration of Competing Interest}

The authors declare no competing financial interest.

\section{Acknowledgements}

This work was financially supported by the National Key Research and Development Program of China (Grant No. 2016YFC1400702), National Natural Science Foundation of China (Grant No. 41776110, 21976209, 21876199), Shandong Provincial Medicine and Health Technology Development Plan (Grant No. 2017WSB290215), Science and Technology Development Plan of Yantai (Grant No. 2017ZH089), Department of Science and Technology of Shandong Province of China (GG201709290055, 2017ZH093), and Taishan Scholar Project Special Funding.

\section{Appendix B. Supplementary data}

Supplementary material related to this article can be found, in the online version, at doi:https://doi.org/10.1016/j.snb.2019.127213.

\section{References}

[1] D.M. Cate, J.A. Adkins, J. Mettakoonpitak, C.S. Henry, Recent developments in paper-based microfluidic devices, Anal. Chem. 87 (2015) 19-41.

[2] X. Li, D.R. Ballerini, W. Shen, A perspective on paper-based microfluidics: current status and future trends, Biomicrofluidics 6 (2012) 011301-011313.

[3] M. Srisa-Art, K.E. Boehle, B.J. Geiss, C.S. Henry, Highly sensitive and rapid detection of salmonella typhimurium using a colorimetric paper based analytical device coupled with immunomagnetic separation, Anal. Chem. 90 (2017) 1035-1043.

[4] W.K. Tomazelli Coltro, C.M. Cheng, E. Carrilho, D.P. de Jesus, Recent advances in low-cost microfluidic platforms for diagnostic applications, Electrophoresis 35 (2014) 2309-2324.

[5] L. Wang, C. Xu, Y. Zhu, Y. Yu, N. Sun, X. Zhang, K. Feng, J. Qin, Human induced pluripotent stem cell-derived beating cardiac tissues on paper, Lab Chip 15 (2015) 4283-4290.

[6] A.K. Yetisen, M.S. Akram, C.R. Lowe, Paper-based microfluidic point-of-care diagnostic devices, Lab Chip 13 (2013) 2210-2251.

[7] J. Ding, B. Li, L. Chen, W. Qin, A three-dimensional origami paper-based device for potentiometric biosensing, Angew. Chem. Int. Edit. 128 (2016) 13227-13231.

[8] X. Sun, B. Li, C. Tian, F. Yu, N. Zhou, Y. Zhan, L. Chen, Rotational paper-based electrochemiluminescence immunodevices for sensitive and multiplexed detection of cancer biomarkers, Anal. Chim. Acta 1007 (2017) 33-39.

[9] S. Wang, L. Ge, X. Song, J. Yu, S. Ge, J. Huang, F. Zeng, Paper-based chemiluminescence ELISA: lab-on-paper based on chitosan modified paper device and waxscreen-printing, Biosens. Bioelectron. 31 (2012) 212-218.

[10] J. Han, A. Qi, J. Zhou, G. Wang, B. Li, L. Chen, Simple way to fabricate novel paperbased valves using plastic comb binding spines, ACS Sens. 3 (2018) 1789-1794.

[11] B. Li, W. Zhang, L. Chen, B. Lin, A fast and low-cost spray method for prototyping and depositing surface-enhanced raman scattering arrays on microfluidic paper based device, Electrophoresis 34 (2013) 2162-2168.

[12] J. Qi, B. Li, X. Wang, L. Fu, L. Luo, L. Chen, Rotational paper-based microfluidic- chip device for multiplexed and simultaneous fluorescence detection of phenolic pollutants based on a molecular-imprinting technique, Anal. Chem. 90 (2018) 11827-11834.

[13] J. Qi, B. Li, X. Wang, Z. Zhang, Z. Wang, J. Han, L. Chen, Three-dimensional paperbased microfluidic chip device for multiplexed fluorescence detection of $\mathrm{Cu}^{2+}$ and $\mathrm{Hg}^{2+}$ ions based on ion imprinting technology, Sens. Actuators B Chem. 251 (2017) $224-233$.

[14] K. Tenda, B. van Gerven, R. Arts, Y. Hiruta, M. Merkx, D. Citterio, Paper-based antibody detection devices using bioluminescent BRET-Switching sensor proteins, Angew. Chem. Int. Edit. 57 (2018) 15369-15373.

[15] J. Hu, S. Wang, L. Wang, F. Li, B. Pingguan-Murphy, T.J. Lu, F. Xu, Advances in paper-based point-of-care diagnostics, Biosens. Bioelectron. 54 (2014) 585-597.

[16] B. Li, L. Yu, Q. Ji, L. Fu, P. Zhang, L. Chen, Controlling capillary-driven fluid transport in paper-based microfluidic devices using a movable valve, Anal. Chem. 89 (2017) 5708-5713.

[17] B. Li, Z. Zhang, J. Qi, N. Zhou, S. Qin, J. Choo, L. Chen, Quantum dot-based molecularly imprinted polymers on three-dimensional origami paper microfluidic chip for fluorescence detection of phycocyanin, ACS Sens. 2 (2017) 243-250.

[18] Y. Zhang, P. Zuo, B.C. Ye, A low-cost and simple paper-based microfluidic device for simultaneous multiplex determination of different types of chemical contaminants in food, Biosens. Bioelectron. 68 (2015) 14-19.

[19] A.W. Martinez, S.T. Phillips, M.J. Butte, G.M. Whitesides, Patterned paper as a platform for inexpensive, low-volume, portable bioassays, Angew. Chem. 46 (2007) 1318-1320.

[20] A.W. Martinez, S.T. Phillips, E. Carrilho, H. Sindi, G.M. Whitesides, Simple telemedicine for developing regions: camera phones and paper-based microfluidic devices for real-time, off-site diagnosis, Anal. Chem. 80 (2008) 3699-3707.

[21] E. Carrilho, A.W. Martinez, G.M. Whitesides, Understanding wax printing: a simple micropatterning process for paper-based microfluidics, Anal. Chem. 81 (2009) 7091-7095.

[22] Y. Lu, W. Shi, L. Jiang, J. Qin, B. Lin, Rapid prototyping of paper-based microfluidics with wax for low-cost, portable bioassay, Electrophoresis 30 (2009) 1497-1500.

[23] T. Songjaroen, W. Dungchai, O. Chailapakul, C.S. Henry, W. Laiwattanapaisal, Blood separation on microfluidic paper-based analytical devices, Lab Chip 12 (2012) 3392-3398.

[24] H. Wang, Y. Li, J. Wei, J. Xu, Y. Wang, G. Zheng, Paper-based three-dimensional microfluidic device for monitoring of heavy metals with a camera cell phone, Anal Bioanal. Chem. 406 (2014) 2799-2807.

[25] K. Yamada, T.G. Henares, K. Suzuki, D. Citterio, Paper-based inkjet-printed microfluidic analytical devices, Angew. Chem. Int. Edit. 54 (2015) 2-19.

[26] W. Dungchai, O. Chailapakul, C.S. Henry, A low-cost, simple, and rapid fabrication method for paper-based microfluidics using wax screen-printing, Analyst 136 (2011) 77-82.

[27] P.D. Haller, C.A. Flowers, M. Gupta, Three-dimensional patterning of porous materials using vapor phase polymerization, Soft Matter 7 (2011) 2428-2432.

[28] X. Li, J. Tian, T. Nguyen, W. Shen, Paper-based microfluidic devices by plasma treatment, Anal. Chem. 80 (2008) 9131-9134.

[29] X. Li, J. Tian, W. Shen, Progress in patterned paper sizing for fabrication of paperbased microfluidic sensors, Cellulose 17 (2010) 649-659.

[30] Y.S. Kim, Y. Yang, C.S. Henry, Laminated and infused Parafilm ${ }^{\oplus}$-paper for paperbased analytical devices, Sens. Actuators B Chem. 255 (2018) 3654-3661.

[31] P. Spicarmihalic, B. Toley, J. Houghtaling, T. Liang, P. Yager, E. Fu, $\mathrm{CO}_{2}$ laser cutting and ablative etching for the fabrication of paper-based devices, $\mathrm{J}$. Micromech. Microeng. 23 (2013) 067003-067008.

[32] J. Nie, Y. Zhang, L. Lin, C. Zhou, S. Li, L. Zhang, J. Li, Low-cost fabrication of paperbased microfluidic devices by one-step plotting, Anal. Chem. 84 (2012) 6331-6335.

[33] D.A. Bruzewicz, R. Meital, G.M. Whitesides, Low-cost printing of poly(dimethylsiloxane) barriers to define microchannels in paper, Anal. Chem. 80 (2008) 3387-3392.

[34] J. Wang, M.R. Monton, X. Zhang, C.D. Filipe, R. Pelton, J.D. Brennan, Hydrophobic sol-gel channel patterning strategies for paper-based microfluidics, Lab Chip 14 (2014) 691-695.

[35] T. Nurak, N. Praphairaksit, O. Chailapakul, Fabrication of paper-based devices by lacquer spraying method for the determination of nickel (II) ion in waste water, Talanta 114 (2013) 291-296.

[36] T.M.G. Cardoso, F.R. de Souza, P.T. Garcia, D. Rabelo, C.S. Henry, W.K.T. Coltro, Versatile fabrication of paper-based microfluidic devices with high chemical resistance using scholar glue and magnetic masks, Anal. Chim. Acta 974 (2017) 63-68.

[37] F. Deiss, W.L. Matochko, N. Govindasamy, E.Y. Lin, R. Derda, Flow-through synthesis on teflon-patterned paper to produce peptide arrays for cell-based assays, Angew. Chem. Int. Ed. 53 (2014) 1-5.

[38] M.M. El-Molla, Synthesis of polyurethane acrylate oligomers as aqueous UV-curable binder for inks of ink jet in textile printing and pigment dyeing, Dyes Pigments 74 (2007) 371-379.

[39] K. Li, Y. Shen, G. Fei, H. Wang, J. Li, Preparation and properties of castor oil/ pentaerythritol triacrylate-based UV curable waterborne polyurethane acrylate, J. Prog. Org. Coat. 78 (2015) 146-154.

[40] C. Lv, L. Hu, Y. Yang, H. Li, C. Huang, X. Liu, Waterborne UV-curable polyurethane acrylate/silica nanocomposites for thermochromic coatings, RSC Adv. 5 (2015) 25730-25737.

[41] Y. Yong, L. Bo, G. Li, S. Jiang, S. Fang, Synthesis and properties of photosensitive silicone-containing polyurethane acrylate for leather finishing agent, Ind. Eng. Chem. Res. 53 (2014) 564-571.

[42] A. Fonseca, Ivo M. Raimundo Jr., J.J.R. Rohwedder, L.O.S. Ferreira, Construction 
and evaluation of a flow injection micro-analyser based on urethane-acrylate resin, Anal. Chim. Acta 603 (2007) 159-166.

[43] L.N.N. Nóbrega, L.D.O. Magalhães, A. Fonseca, A urethane-acrylate microflowanalyzer with an integrated cadmium column, Microchem. J. 110 (2013) 553-557.

[44] T.R. Sampaio, A. Fonseca, Chemiluminescence detection in urethane-acrylate microfluidic devices, Sens. Actuators B Chem. 213 (2015) 215-221.

[45] V. Rajendra, C. Sicard, J.D. Brennan, M.A. Brook, Printing silicone-based hydrophobic barriers on paper for microfluidic assays using low-cost ink jet printers, Analyst 139 (2014) 6361-6365.

[46] B. Li, L. Fu, W. Zhang, W. Feng, L. Chen, Portable paper-based device for quantitative colorimetric assays relying on light reflectance principle, Electrophoresis 35 (2014) 1152-1159.

[47] S.M.Z. Hossain, C. Ozimok, C. Sicard, S.D. Aguirre, M.M. Ali, Y. Li, J.D. Brennan, Multiplexed paper test strip for quantitative bacterial detection, Anal. Bioanal. Chem. 403 (2012) 1567-1576.

Dong Lin is currently a PhD degree candidate, under the guidance of Prof. Lingxin Chen, at Yantai Institute of Coastal Zone Research, Chinese Academy of Sciences, since 2019. He received his master degree at School of Chemistry and Chemical Engineering, University of Jinan, in 2011. In the same year, he joined in School of Pharmacy, Binzhou Medical University, as an assistant engineer. In 2015, she became an engineer. His current research interests focus on the fabrication of novel microfluidic paper-based analytical devices and their application in biological analysis.

Bowei Li is currently as an associate professor at Yantai Institute of Coastal Zone Research, Chinese Academy of Sciences. He received his $\mathrm{PhD}$ at Dalian Institute of Chemical Physics, Chinese Academy of Sciences, in 2009. During 2009-2011, he worked at Department of Chemistry, Florida State University. In 2011, he joined in Yantai Institute of Coastal Zone Research, Chinese Academy of Sciences, as an assistant professor. In 2017, he became an associate professor. His research interests focus on the environmental monitoring, point-of-care testing, and other fields of basic research on chemical and biological using microfluidic chip platform.

Ji Qi is currently a PhD degree candidate, under the guidance of Prof. Liqiang Luo and Prof. Lingxin Chen, at College of Sciences, Shanghai University, China, since 2017. He received his master degree, under the guidance of Prof. Lingxin Chen and Prof. Zhuo
Wang, at School of Environment and Materials Engineering, Yantai University and Yantai Institute of Coastal Zone Research, Chinese Academy of Sciences, since 2015. His current research interests are the using imprinting materials to make investigations on the paperbased microfluidic devices.

Xiaofei Ji is currently as an associate professor at School of Pharmacy, Binzhou Medical University. He received her PhD at School of Life Sciences, Shandong University, in 2013. In the same year, he joined in School of Pharmacy, Binzhou Medical University, as an assistant professor. In 2017, he became an associate professor. His research interests are focused on new detection techniques for foodborne pathogens.

Shixuan Yang received her BS degree from School of Food Science and Engineering, Shandong Agriculture and Engineering University, in 2018. She is studying for her master degree in School of Food Science and Engineering, Shandong Agricultural University, Joint-Educated at Yantai Institute of Coastal Zone Research, Chinese Academy of Sciences. Her current research interest is molecularly imprinted polymers based research for environmental analysis.

Wenhai Wang is currently as a senior engineer at Yantai Institute of Coastal Zone Research, Chinese Academy of Sciences. He received his $\mathrm{PhD}$ in organic chemistry from the College of Chemistry of Sichuan University, Chengdu, in 2009. In the same year, he joined in Yantai Institute of Coastal Zone Research, Chinese Academy of Sciences, as an assistant professor. In 2015, he became a senior engineer. His current research interest focuses on the design and synthesis of surface-enhanced Raman scattering probes in vivo Raman imaging.

Lingxin Chen is currently as a professor at Yantai Institute of Coastal Zone Research, Chinese Academy of Sciences. He received his $\mathrm{PhD}$ degree in analytical chemistry from the Dalian Institute of Chemical Physics, Chinese Academy of Sciences, Dalian, in 2003. After 2 years of postdoctoral experience at the Department of Chemistry, Tsinghua University, Beijing, he joined first as a BK21 researcher and then as a research professor at the Department of Applied Chemistry, Hanyang University, Korea, in 2006. In 2009, as a professor, he joined the Yantai Institute of Coastal Zone Research, Chinese Academy of Sciences, Yantai. His research interests include the studies of novel properties of materials such as functionalized nanoparticles for developing nanoscale biochemical analysis methods and molecular imprinting-based sample pretreatment technology. 